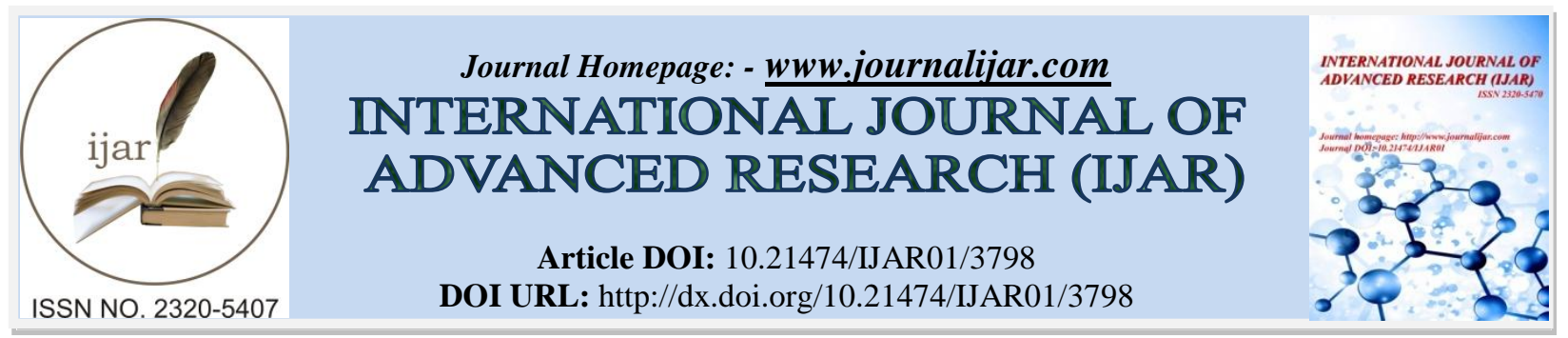

RESEARCH ARTICLE

\title{
IMPACT OF QRS COMPLEX FRAGMENTATION ON HOSPITAL OUTCOME OF PATIENTS UNDERGOING PRIMARY PERCUTANEOUS CORONARY INTERVENTION.
}

Hatem Badawi*, Ragab mahfouz, Tamer Moustafa and Marwa Gad.

Cardiology Department, Faculty of Medicine-Zagazig University, Egypt.

\section{Manuscript Info}

Manuscript History

Received: 15 February 2017

Final Accepted: 06 March 2017

Published: April 2017

\section{Abstract}

Background and Aim: Fragmented QRS (fQRS) was linked to myocardial ischemia and scar. The aim of this study was to investigate the impact of fQRS on the hospital outcome and major adverse cardiac events (MACE) in patients with acute STEMI treated with primary percutaneous coronary intervention (PCI).

Methods: The present study was conducted on 90 patients with acute STEMI and treated with primary PCI, in Zagazig University Hospital in Egypt, during the period from January 2015 to May 2016. Careful history taking, clinical data, full lab, left ventricular ejection fraction calculated by biplane Simpson method and angiographic findings were taken. ECG records were obtained on admission and 2 hours after primary PCI. During hospitalization, ventricular fibrillation (VF) and ventricular tachycardia (VT), cardiogenic shock, cardiopulmonary resuscitation, re-infarction, and death were regarded as MACE. We divided the patients into 2 groups as regard fQRS on admission ECG into fragmented $(n=40)$ group and non-fragmented $(n=50)$ group.

Results: There was significant statistical difference regarding inhospital AF, being more frequent in fQRS group (P value 0.049). There was significant statistical difference regarding LVEF, being lower in fQRS group (P value 0.009). There was significant statistical difference regarding the incidence of three vessel disease, being more frequent in fQRS group (P value 0.02). There was significant statistical difference regarding culprit lesion being proximal, being more frequent in fQRS group ( $\mathrm{p}$ value 0.009). By multivariate analysis, we found that fQRS was a predictor for unfavorable hospital outcome (P value 0.046)

Conclusions: Assessment of fQRS by ECG is a simple, widely available, and noninvasive modality that may be useful to predict hospital outcome, incidence of proximal and three vessels disease in patients with acute STEMI undergoing primary PCI.

Copy Right, IJAR, 2017,. All rights reserved.

\section{Introduction:-}

Acute myocardial infarction (AMI) is due to occlusion in the coronary artery that leads to severe ischemia and necrosis of myocardium resulting in high mortality rate and poor prognosis. The primary causes of death are arrhythmia and heart failure (1). Fragmented QRS complexes (fQRS) are frequently seen on the 12 leads electrocardiograms (ECG) with a narrow or wide QRS complex. FQRS was defined as an additional R wave (R') or 
notching within the QRS complex $(2,3)$. Heart failure is commonly seen in patients with a large area of myocardial infarction $(>40 \%)$ and sometimes results in cardiogenic shock. FQRS has been proved to be valuable tool for predicting the incidence of malignant cardiac arrhythmia and left ventricular systolic dysfunction (4).The presence of fQRS on ECG is associated with myocardial scarring, ischemia, and fibrosis and originates from deterioration in the process of signal transduction and ventricular depolarization (5). The presence of fQRS in coronary artery disease (CAD) patients is known to be significantly associated with MACE, left ventricular dysfunction, and impairment of myocardial perfusion $(6,7)$. FQRS originates from small abnormal myocardial areas where ventricular activation is delayed and asynchronous. Partial damage of the conducting system inside the ventricle causes the notching of the QRS segment on the ECG (8).

\section{Materials and Methods:-}

This study was carried out in cardiology department, Zagazig university hospital in Egypt from January 2015 to May 2016. The study included (90) patients admitted with acute STEMI undergoing primary PCI. Informed consent was obtained from every patient on participation of the study. Inclusion criteria; patients diagnosed with acute STEMI and treated with primary PCI. For acute STEMI, the following diagnostic criteria were used: 1) ST segment elevation in $\geq 2$ consecutive derivations (in chest derivations $\geq 2 \mathrm{~mm}$, extremity derivations $\geq 1 \mathrm{~mm}$ ), 2) Ischemic type chest pain lasting more than 30 minutes, 3) A 2 fold or greater elevation in serum creatine phosphokinase myocardial band (CKMB) and troponin levels. Exclusion criteria: patients with significant organic valvular heart disease, patients with congenital heart disease, patients with bundle branch block (LBBB, incomplete or complete RBBB or duration QRS $>120 \mathrm{~ms}$, Patients with known history of coronary heart disease, patients with permanent pacemakers and patients with renal impairment. All patients were subjected to the following: History evaluation for hypertension, diabetes mellitus, smoking history, family history for CAD, and hyperlipidemia. Hypertension was defined as use of antihypertensive drugs or documented blood pressure reading greater than 140/90 $\mathrm{mmHg}$. Diabetes mellitus was defined as use of antidiabetic drugs or insulin or fasting blood glucose levels over $126 \mathrm{~m} / \mathrm{dL}$ or over $200 \mathrm{mg} / \mathrm{dL}$ at any measurement. Patients who were using tobacco on admission or had quit smoking within the last year were considered as smokers. The family history for CAD was defined as history of documented CAD or sudden cardiac death in first degree relative before age of 55 for males and 65 for females. Clinical examination including: heart rate, arterial blood pressure, oxygen saturation, Killip classification. Electrocardiographic examination: on admission and 2 hours after PCI. The determination of QRS complex fragmentation by ECG records (Filter range $0.5 \mathrm{~Hz}$ to $150 \mathrm{~Hz}, \mathrm{AC}$ filter $60 \mathrm{~Hz}$, at a speed of $25 \mathrm{~mm} / \mathrm{s}$ and an amplitude of $10 \mathrm{~mm} / \mathrm{mV}$ ) and fQRS complexes on a 12 lead ECG were defined as various RSR' patterns ( $\geq 1 R^{\prime}$ or notching of $S$ wave or R wave) with or without $Q$ waves lacking typical $\mathrm{BBB}$ in 2 contiguous leads corresponding to major coronary artery territory. Echocardiography: left ventricular systolic ejection fraction (LVEF) was measured by the modified Simpson method. Emergency coronary angiography was performed through the femoral artery. Following femoral artery puncture, all patients were given an intravenous bolus of $100 \mathrm{U}$ per $\mathrm{kg}$ of unfractionated heparin. According to the lesion type, primary PCI was performed only to the infarct related artery (IRA). Success in the acute phase was defined as less than 50\% residual narrowing with TIMI II or TIMI III flow achieved in the IRA for each procedure. Following angioplasty, all patients were admitted to the coronary intensive care unit and were given $1 \mathrm{mg} / \mathrm{kg}$ of subcutaneous enoxaparin twice a day, daily $81 \mathrm{mg}$ aspirin, and $150 \mathrm{mg}$ clopidogrel. While the choice of Glycoprotein $\mathrm{IIb} / \mathrm{III}$ inhibitor treatment was left up to the primary physician, beta blockers, ACE inhibitors or ARBs, and statins were given according to the guidelines. Patients who were not candidate for primary PCI and need emergency coronary artery bypass surgery were ruled out from the study. During the hospitalization follow up of patients, VT, VF, cardiogenic shock, cardiopulmonary resuscitation (CPR), re-infarction, stroke, and death were regarded as major adverse cardiac events (MACE).The patients were divided according to QRS complex fragmentation on ECG upon admission into 2 groups: fragmented QRS (FQRS) Group (40 patients) and non-fragmented QRS (Non-FQRS) Group (50 patients).

\section{Statistical Analysis:-}

The following Statistical tests and parameters were used: Mean is the sum of the values, $\mathrm{N}$ is the number of subjects. Standard deviation (SD) is the sum of the square of the differences of each observation from the mean. The pairedsamples $\mathrm{T}$ test compares the means of two variables for a single group. A chi-square test used to see if there was a relationship between two categorical variables (association between qualitative variables). Pearson correlation coefficient is a measure of the linear correlation (dependence) between two variables $\mathrm{X}$ and $\mathrm{Y}$, giving a value between +1 and -1 inclusive, where 1 is total positive correlation, 0 is no correlation, and -1 is total negative correlation. One-way analysis of variance (ANOVA) is a technique used to compare means of two or more samples. The threshold of significance was fixed at 5\% level (P value) Significance was detected according to P-value as 
follow: $\mathrm{P}>0.05=$ Non-significant, $\mathrm{P}<0.05=$ significant and $\mathrm{P}<0.001=$ highly significant. Continuous variables were expressed as mean \pm SD. Categorical variables were expressed as numbers and percentages. A chi-square test used to see a relationship between two categorical variables (association between qualitative variables). The pairedsamples $\mathrm{T}$ test procedure was used to compare the means between the two groups. One-way analysis of variance (ANOVA) test was used for comparison. The correlation is done using Pearson correlation. All was calculated using SPSS version 19-software program.

\section{Results:-}

There was significant statistical difference between the 2 groups regarding the incidence of AF and LVF (P value 0.049 and 0.005 respectively), being more frequent in the fQRS group. There was significant difference between 2 groups regarding LVEF, being lower in fQRS group (P value 0.009). There was significant statistical difference between the two groups regarding single, two and three vessel disease ( $P$ value 0.02$)$, incidence of three vessel disease being higher in fQRS group. There was significant statistical difference regarding culprit lesion being proximal, being more frequent in fQRS group ( $\mathrm{p}$ value 0.009). There was significant statistical difference between the 2 groups regarding TIMI score of the culprit artery and myocardial blush grade (MBG) after primary PCI (P value 0.047 and 0.039 respectively), being higher in the non-FQRS complex group. The results showed that fQRS was significantly correlated with both myocardial blush post-PCI and TIMI score post-PCI $(\mathrm{p}$ value <0.05), meanwhile it had a highly significant correlation with proximal lesion, three vessel disease and LVEF ( $p$ value $<0.01$, $<0.001,<0.01$ respectively). By multivariate analysis, we found that fQRS was a predictor for unfavorable hospital outcome (P value 0.046). Sensitivity and specificity of fQRS to predict unfavorable outcome: Sensitivity $78.6 \%$ and Specificity $61.8 \%$, with positive predictive value $27.5 \%$ and negative predictive value $94 \%$.

Table 1: Angiographic data in study groups.

\begin{tabular}{|c|c|c|c|c|c|c|}
\hline \multicolumn{2}{|l|}{ Variables } & \multicolumn{2}{|c|}{$\begin{array}{l}\text { FQRS } \\
(\mathrm{n})=40\end{array}$} & \multicolumn{2}{|c|}{$\begin{array}{l}\text { Non-FQRS } \\
(\mathrm{n})=50\end{array}$} & \multirow[t]{2}{*}{ P-Value } \\
\hline & & $\mathrm{n}$ & $\%$ & $\mathrm{n}$ & $\%$ & \\
\hline \multirow{3}{*}{$\begin{array}{l}\text { Total number of significant diseased } \\
\text { vessels }\end{array}$} & one & 15 & 37.5 & 30 & 60 & \multirow[t]{3}{*}{$0.02 *$} \\
\hline & Two & 10 & 25 & 13 & 26 & \\
\hline & Three & 15 & 37.5 & 7 & 14 & \\
\hline \multirow{3}{*}{ Culprit artery } & LAD & 21 & 52.5 & 22 & 44 & \multirow[t]{3}{*}{0.46} \\
\hline & LCX & 8 & 20 & 8 & 16 & \\
\hline & RCA & 11 & 27.5 & 20 & 40 & \\
\hline \multicolumn{2}{|l|}{ Culprit lesion is proximal } & 23 & 57.5 & 15 & 30 & $0.009 *$ \\
\hline \multirow[t]{4}{*}{ TIMI score before PPCI } & 0 & 26 & 65 & 30 & 60 & \multirow[t]{4}{*}{0.63} \\
\hline & 1 & 5 & 12.5 & 6 & 12 & \\
\hline & 2 & 9 & 22.5 & 12 & 24 & \\
\hline & 3 & 0 & 0 & 2 & 4 & \\
\hline \multirow[t]{2}{*}{ TIMI score after PPCI } & 2 & 5 & 12.5 & 1 & 2 & \multirow[t]{2}{*}{$0.047 *$} \\
\hline & 3 & 35 & 87.5 & 49 & 98 & \\
\hline \multirow[t]{3}{*}{ MBG after PPCI } & 1 & 2 & 5 & 0 & 0 & \multirow[t]{3}{*}{$0.039 *$} \\
\hline & 2 & 12 & 30 & 7 & 14 & \\
\hline & 3 & 26 & 65 & 43 & 86 & \\
\hline
\end{tabular}

Table 2:- Complication distribution between study groups.

\begin{tabular}{|l|l|l|l|l|l|}
\hline \multirow{2}{*}{ Variables } & \multicolumn{2}{l|}{$\begin{array}{l}\text { FQRS } \\
\text { (n)=40n-FQRS }\end{array}$} & \multirow{2}{*}{$\begin{array}{l}\text { P-Value } \\
(\mathrm{n})=50\end{array}$} \\
\cline { 2 - 5 } & $\mathrm{n}$ & $\%$ & $\mathrm{n}$ & $\%$ & \\
\hline AF & 3 & 7.5 & 0 & 0 & $0.049^{*}$ \\
\hline VT & 3 & 7.5 & 2 & 4 & 0.47 \\
\hline VF & 1 & 2.5 & 1 & 2 & 0.87 \\
\hline Ischemic Stroke & 2 & 5 & 0 & 0 & 0.11 \\
\hline Cardiogenic Shock & 3 & 7.5 & 1 & 2 & 0.208 \\
\hline LVF & 8 & 20 & 1 & 2 & $0.005^{*}$ \\
\hline Cardio-pulmonary arrest & 1 & 2.5 & 1 & 2 & 0.87 \\
\hline Mortality & 1 & 2.5 & 1 & 2 & 0.87 \\
\hline
\end{tabular}


Table 3: Correlation of fragmented QRS complex to different variables.

\begin{tabular}{|l|c|c|c|}
\hline Variables & $\mathrm{r}$ & OR (95\% C.I) & P value \\
\hline MBG post PCI & 0.399 & $2.79(1.73-3.82)$ & $<0.05$ \\
\hline TIMI score post PCI & 0.425 & $2.93(1.99-3.95)$ & $<0.05$ \\
\hline Proximal vessel disease & 0.511 & $4.32(1.82-7.22)$ & $<0.01$ \\
\hline Three vessels disease & 0.659 & $1.33(0.601-1.92)$ & $<0.001$ \\
\hline AF & 0.158 & $1.33(0.601-1.92)$ & $>0.05$ \\
\hline LVEF (\%) & 0.491 & $3.21(1.23-5.36)$ & $<0.01$ \\
\hline
\end{tabular}

Table 4: Multivariate logistic regression analysis for predictors of hospital outcome

\begin{tabular}{|l|l|l|l|l|l|}
\hline & Wald & P & \multirow{2}{*}{ OR } & \multicolumn{2}{l|}{ 95\% C.I. for EXP(B) } \\
\cline { 5 - 6 } & & & & Lower & Upper \\
\hline HTN & 1.632 & 0.201 & 0.332 & 0.061 & 1.803 \\
\hline TIMI score after PPCI & 0.176 & 0.674 & 1.819 & 0.112 & 29.656 \\
\hline Myocardial Blush grade after PPCI & 1.099 & 0.295 & 0.437 & 0.093 & 2.053 \\
\hline QRS complex Fragmentation & 3.868 & $0.046^{*}$ & 4.675 & 1.102 & 17.207 \\
\hline
\end{tabular}

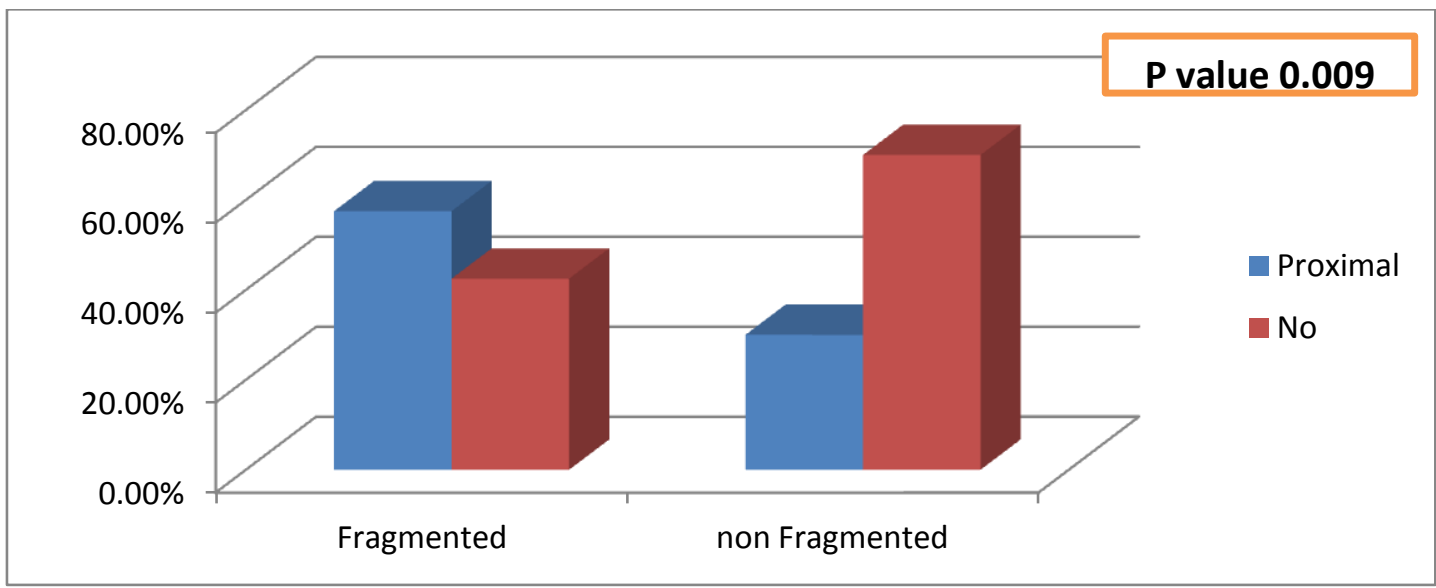

Figure 1:- Culprit lesion being proximal in study groups.

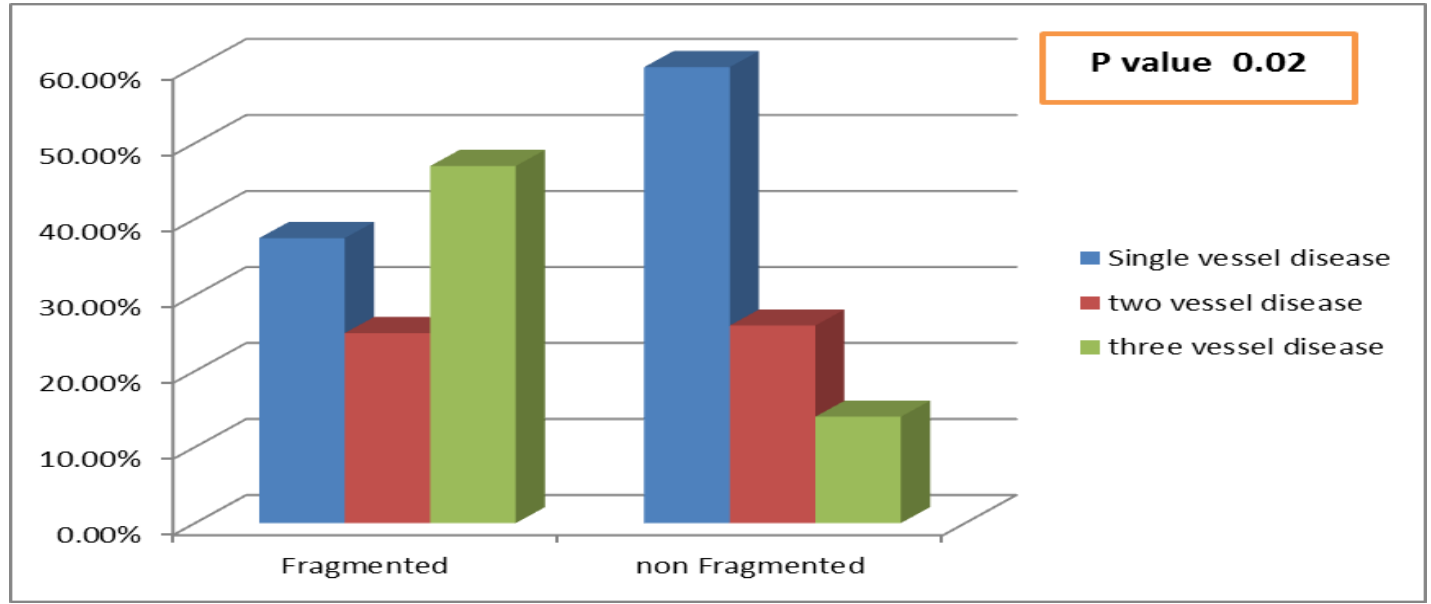

Figure 2:- Incidence of single, two vessel disease and three vessel disease in study groups 


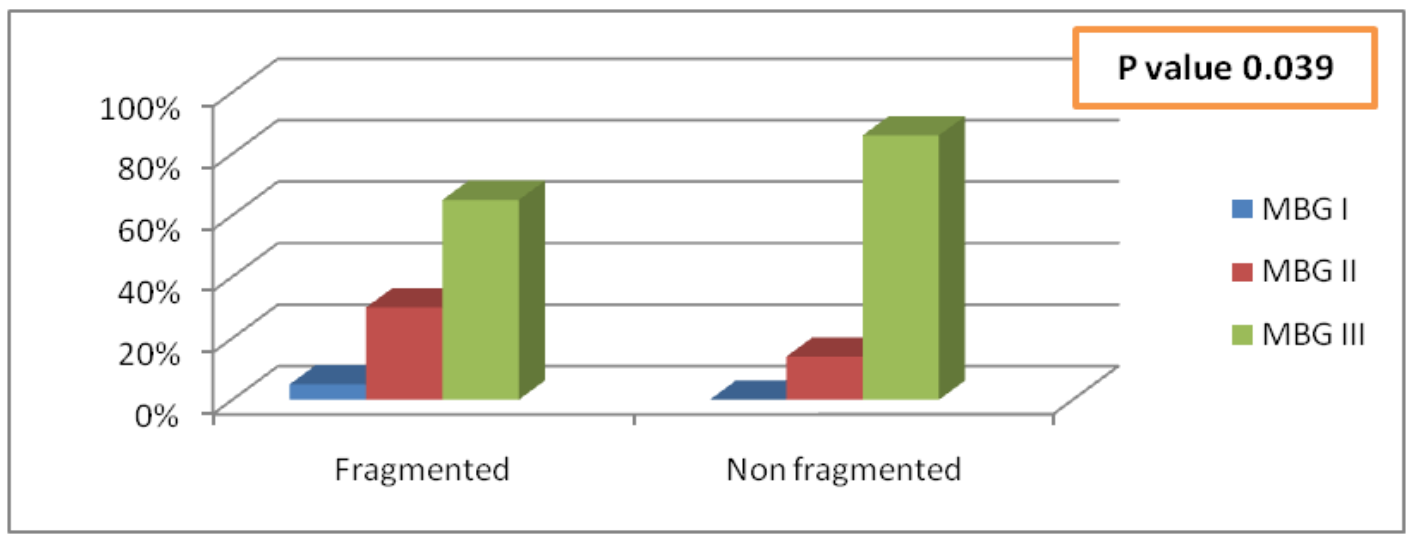

Figure 3: Myocardial blush grade (MBG) after primary PCI in study groups.
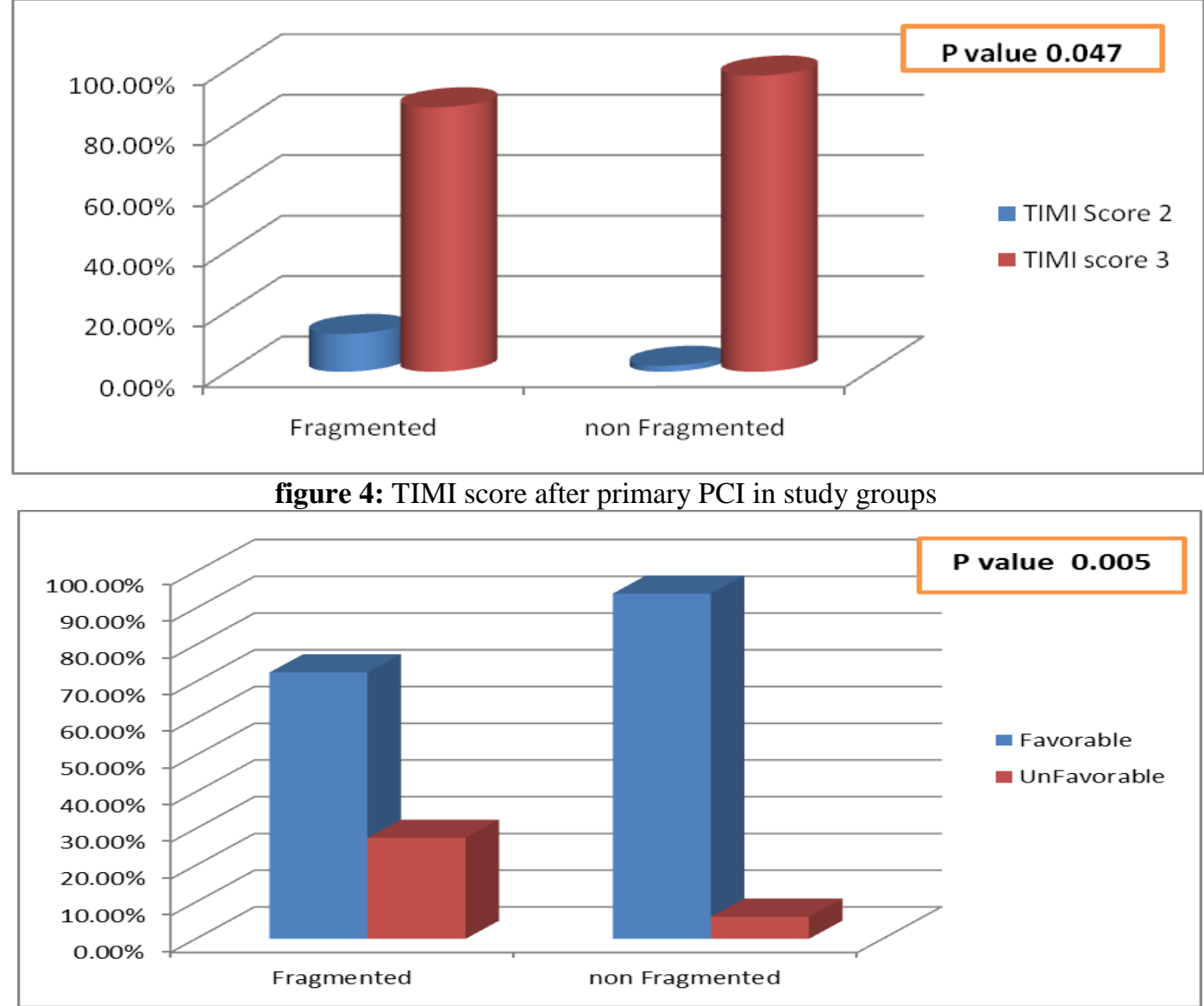

Figure 5:- Favorable and unfavorable outcome in study groups.

\section{Discussion:-}

Our study showed no significant statistical difference between the 2 groups regarding number of patients developed in-hospital MACE. This was discordant with Yildirım E et al 2014 who found statistical significant difference between the two groups regarding number of patients developed in-hospital MACE, being higher in fQRS group, this discrepancy could be explained by large sample volume included in their study and also they included patients with wide QRS complex, renal impairment and past history of IHD. Our study showed significant statistical difference between the two groups regarding number of patients developed in-hospital AF, being more frequent in fQRS group. This was discordant Uslu N et al 2015 who found that in-hospital AF occurred in 5 patients with fQRS (3.3\%) versus 14 patients in non-fQRS (3.6\%) with $\mathrm{p}$ value 0.850 . Our study showed significant statistical difference between the two groups regarding number of patients developed left ventricular heart failure (LVF), 
being more frequent in fQRS group. This was concordant Uslu $\mathrm{N}$ et al 2015, who found that advanced heart failure occurred in $17.6 \%$ of patients in fQRS group versus $6.9 \%$ of patients in non-fQRS group, $\mathrm{P}$ value $<0.001$. In our study, there was significant difference between 2 groups regarding LVEF, being lower in fQRS group. This was concordant with Yildırım E et al 2014, who found that LVEF (\%) 50.21 10.47 (50) in non-fQRS group versus $41.66 \pm 11.45$ (40) in fQRS group (P value 0.001). In our study, there was significant statistical difference between the two groups regarding incidence of three vessel diseases, being more frequent in fQRS group. This was concordant with Tanriverdi $\mathrm{Z}$ et al 2015, who found that incidence three vessels disease (\%) was 56 (61.5) in fQRS group versus 23 (14.6) in non-fQRS group, p value <0.001. In our study, there was significant statistical difference regarding culprit lesion being proximal, being more frequent in fQRS group. This was concordant with Tanriverdi $\mathrm{Z}$ et al 2015 who found that the incidence of proximal vascular disease (\%) was 74 (81.3) in fQRS group versus 60 (38.2) in non-fQRS group, $\mathrm{p}$ value $<0.001$. In our study, there was significant statistical difference between the 2 groups as regard TIMI score of the culprit artery after primary PCI, being higher in the non-fQRS group. This was discordant with Cetin M et al.,2012 who found that post-PCI TIMI score (2 and 3) was detected in $90 \%$ of patients with fQRS versus $86 \%$ of patient with non-fQRS, p value $>0.05$. In our study, there was significant statistical difference between the 2 groups as regard myocardial blush grade after primary PCI, being higher in the non-FQRS complex group. This was concordant with Cetin M et al., 2012, who stated that incidence of post-PCI blush score (2 and 3) was detected in $81 \%$ of patients in non-fQRS group versus $48 \%$ in fQRS group, p value $<0.001$. In our study, results showed that fQRS was significantly correlated with both myocardial blush post-PCI and TIMI score post-PCI ( $\mathrm{p}$ value $<0.05$ ), meanwhile it had a highly significant correlation with proximal lesion, three vessel disease and LVEF ( $\mathrm{p}$ value $<0.01,<0.001,<0.01$ respectively). By multivariate analysis, we found that fQRS was a predictor for unfavorable hospital outcome (P value 0.046). Sensitivity and specificity of fQRS complex to predict unfavorable outcome: Sensitivity $78.6 \%$ and Specificity $61.8 \%$, with positive predictive value $27.5 \%$ and negative predictive value $94 \%$.

\section{Limitations:-}

Limitations of our study were: Small sample size of our study this could have biased our results; our study was applied on STEMI patients only excluding those with NSTEMI and unstable angina and absence of long-term follow-up.

\section{Conclusion:-}

The assessment of fQRS by surface ECG is a simple, widely available, and noninvasive modality that may be useful for identifying patients at higher cardiac risk and predicting in-hospital cardiac events. FQRS may be used as a simple predictive parameter to predict in-hospital AF, LVF, proximal vessel disease and three vessels disease in patients with acute STEMI undergoing primary PCI.

\section{References:-}

1. Wang Y and Zhao HN. Investigation of changing and significance of connexin43, $[\mathrm{Ca} 2+]$ and apoptosis in early phase of acute myocardial ischemia. Chin J Cardiac Pacing Electrophysiol. 2011; 4(25):349-352.

2. Das MK. Significance of a fragmented QRS complex versus a Q wave in patients with coronary artery disease. Circulation. 2006; 113:2495.

3. Das MK. Fragmented wide QRS on a 12-lead ECG: a sign of myocardial scar and poor prognosis. Circ Arrhythm Electrophysiol. 2008; 1:258.

4. Cheema A, Khalid A, Wimmer A, et al. Fragmented QRS and mortality risk in patients with left ventricular dysfunction. Circ Arrhythm Electrophysiol. 2010; 3(4):339-344.

5. Pietrasik G and Zareba W. QRS Fragmentation: Diagnostic and prognostic significance. Cardiol J. 2012; 19(2):114-21.

6. Das MK. Fragmented QRS on a 12-lead ECG: a predictor of mortality and cardiac events in patients with coronary artery disease. Heart Rhythm. 2007; 4:1385.

7. Korhonen P. Fragmented QRS in prediction of cardiac deaths and heart failure hospitalizations after myocardial infarction. Ann Noninvasive Electrocardiol. 2010; 15:130 .

8. Bayes de Luna A. Electrographic patterns of ischemia, injury and necrosis. Clinical electrocardiography: A textbook. 2nd ed. Futura Publishing Company, Inc.; Armonk, NY: 1998. pp. 141-42.

9. Ma X, Duan W, Poudel P, et al. Fragmented QRS complexes have predictive value of imperfect ST-segment resolution in patients with STEMI after primary percutaneous coronary intervention. Am J Emerg Med. 2016 Mar; 34(3):398-402. 
10. Yıldırım E, Karaçimen D, Ozcan KS, et al. The relationship between fragmentation on electrocardiography and in-hospital prognosis of patients with acute myocardial infarction. Med Sci Monit. 2014 Jun 3; 20:913-9.

11. Cetin M, Kocaman SA, Kiris T, et al. Absence and resolution of fragmented QRS predict reversible myocardial ischemia with higher Probability of ST segment resolution in patients with ST segment elevation myocardial infarction. Korean Circ J. 2012 Oct; 42(10):674-83.

12. Uslu N, Gul M, Cakmak HA, et al. The assessment of relationship between fragmented QRS complex and left ventricular wall motion score index in patients with ST elevation myocardial infarction who underwent primary percutaneous coronary intervention. Ann Noninvasive Electrocardiol. 2015 Mar; 20(2):148-57.

13. Tanriverdi Z, Dursun H, Simsek MA, et al. The Predictive Value of Fragmented QRS and QRS Distortion for High-Risk Patients with STEMI and for the Reperfusion Success. Ann Noninvasive Electrocardiol. 2015 Nov; 20(6):578-85. 